\title{
BMJ Open Efficacy and safety of super selective bronchial artery coil embolisation for haemoptysis: a single-centre retrospective observational study
}

Hideo Ishikawa, ${ }^{1}$ Masahiko Hara, ${ }^{2}$ Misaki Ryuge, ${ }^{1}$ Jun Takafuji, ${ }^{1}$ Mihoko Youmoto, ${ }^{1}$ Masanori Akira, ${ }^{3}$ Yukio Nagasaka, ${ }^{4}$ Daijiro Kabata, ${ }^{2}$ Kouji Yamamoto, ${ }^{2}$ Ayumi Shintani $^{2}$

To cite: Ishikawa $\mathrm{H}$, Hara M, Ryuge $\mathrm{M}$, et al. Efficacy and safety of super selective bronchial artery coil embolisation

for haemoptysis: a singlecentre retrospective observational study. BMJ Open 2017;7:e014805. doi:10.1136/bmjopen-2016014805

- Prepublication history and additional material is available. To view please visit the journal (http://dx.doi.org/ 10.1136/bmjopen-2016014805).

Received 20 October 2016 Revised 20 December 2016 Accepted 19 January 2017

CrossMark

For numbered affiliations see end of article.

Correspondence to Dr Masahiko Hara; masahiko.hara@dmi.med. osaka-u.ac.jp

\section{ABSTRACT}

Objectives: Evidence on the safety and long-term efficacy of super selective bronchial artery embolisation (SSBAE) using platinum coils in patients with haemoptysis is insufficient. The objective of the present study was to evaluate the safety and the 3-year postprocedure haemoptysis-free survival rate of de novo elective SsBAE using platinum coils rather than particles for the treatment of haemoptysis.

Design: A single-centre retrospective observational study.

Setting: Hemoptysis and Pulmonary Circulation Center in Japan.

Participants: A total of 489 consecutive patients with massive and non-massive haemoptysis who underwent de novo elective SSBAE without malignancy or haemodialysis.

Interventions: SSBAE using platinum coils. All patients underwent CT angiography before the procedure for identifying haemoptysis-related arteries (HRAs) and for procedural planning.

Primary and secondary outcome measures: The composite of the 3-year recurrence of haemoptysis and mortality from the day of the last SsBAE session. Each component of the primary end point and procedural success defined as successful embolisation of all target HRAs were also evaluated.

Results: The median patient age was 69 years, and $46.4 \%$ were men. The total number of target vessels was 4 (quartile 2-7), and the procedural success rate was $93.4 \%$. There were $8(1.6 \%)$ major complications: 1 aortic dissection, 2 symptomatic cerebellar infarctions and 5 mediastinal haematoma cases. The haemoptysis-free survival rates were estimated by the Kaplan-Meier analysis at $86.9 \%(95 \% \mathrm{Cl} 83.7 \%$ to $90.2 \%)$ at 1 year, $79.4 \%(74.8 \%$ to $84.3 \%)$ at 2 years and $57.6 \%(45.1 \%$ to $73.4 \%)$ at 3 years. Although not statistically significant by the adjusted analysis of variance with multiple imputation of missing variables, cryptogenic haemoptysis tended to show the most favourable outcome and non-tuberculous mycobacterium showed the worst outcome (adjusted $\mathrm{p}=0.250$ ).

Conclusions: We demonstrated the safety and longterm efficacy of elective ssBAE using platinum coils

\section{Strengths and limitations of this study}

The strengths of this study include the large sample size and standardiszed protocol of the elective super selective bronchial artery embolisation (ssBAE).

- We could perform ssBAE safely with high success rates.

- Our study is a single-centre, retrospective observational study.

- Detailed information about the status of the underlying respiratory disorders of the patients who had an SSBAE could not be considered in the statistical analysis.

and established that it can be a valuable therapeutic option for treating patients with haemoptysis.

\section{INTRODUCTION}

Haemoptysis is defined as airway bleeding and is a serious clinical complication, which can be fatal. ${ }^{1-6}$ There are essentially four treatment options for haemoptysis with respiratory and haemodynamic supports: drug therapy, bronchoscopic intervention, catheter intervention, such as bronchial artery embolisation (BAE), and lobectomy. ${ }^{1-6}$ BAE has been introduced on the basis of the rationale that one of the main causes of haemoptysis is a systemic-pulmonary shunt, such as a bronchopulmonary shunt, which results in lung vessel rupture $;^{4-8}$ it has played an important role in emergent palliative haemostasis of massive haemoptysis for decades. ${ }^{5}$ Given the advancements in interventional devices and techniques, such as CT angiography, and since persistent sudden unexpected haemoptysis also severely impairs the patients' quality of life, more investigators have recently examined the use of BAE for 
long-term haemostasis. ${ }^{9-14}$ In fact, several reports have demonstrated improvements in long-term prognosis after BAE with polyvinyl alcohol (PVA) or N-butyl-2-cyanoacrylate (NBCA) when compared with gelatin sponges. ${ }^{10-14}$ However, since extreme caution should be exercised when using bloodstream-dependent embolisation materials, such as PVA or NBCA, controllable embolisation materials, such as metallic coils, can also be a candidate material for safe and effective BAE procedures in theory. ${ }^{10-15}$ Since little evidence is available on BAE with metallic coils, the aim of this study was to evaluate the safety and long-term (3-year) efficacy of elective super selective BAE (ssBAE) using platinum coils as the embolisation material with procedural planning based on CT angiography findings in patients with persistent haemoptysis retrospectively; we enrolled 489 consecutive patients at Eishinkai Hemoptysis and Pulmonary Circulation Center (EHPC) in Japan.

\section{METHODS}

\section{Study patients and data collection}

Among the 533 consecutive patients with haemoptysis, who visited the EHPC from April 2010 to December 2015, 489 patients (696 procedures) with available outcome data who underwent de novo elective ssBAE were enrolled in this study. Haemoptysis was defined as airway bleeding with an estimated volume of $>20 \mathrm{cc}$ in each event. In this study, haemoptysis included both massive and non-massive haemoptysis. Approximately $20 \mathrm{~mL}$ of haemoptysis was the minimum required condition for candidates of ssBAE; however, the indication of ssBAE was made in a comprehensive manner, considering how severely haemoptysis impairs the patients' quality of life. Our typical candidates were patients who experienced persistent sudden unexpected haemoptysis, which severely impairs their quality of life regardless of the amount of haemoptysis. For example, most of them might hesitate to go shopping because they are constantly afraid of sudden unexpected haemoptysis in public. Thus, non-massive haemoptysis was also our target. Recurrence of haemoptysis was defined as haemoptysis of at least $20 \mathrm{~mL}$ as defined above, making patients opt to undergo another BAE due to severe impairment of their quality of life, because our goal was to completely eradicate the haemoptysis and because most patients underwent ssBAE for eventless daily life. Since the EHPC is basically an elective ssBAE centre and our objective in this study was to elucidate the efficacy of ssBAE for long-term haemostasis, we excluded emergency ssBAE. The reason for this is that the purpose of ssBAE is totally different between emergency and elective ssBAE at our institution. The main purpose of emergency ssBAE is rapid haemostasis to achieve haemodynamic and respiratory stability leaving aside a long-term efficacy. In contrast, the main purpose of elective ssBAE is to achieve long-term haemostasis to improve the quality of life of patients. Thus, exclusion of emergency ssBAE cases is needed to appropriately evaluate the long-term efficacy of elective ssBAE. Further, 32 patients with lung cancer and 6 patients with haemodialysis were also excluded because the impacts of malignancy or haemodialysis on the survival rate were assumed to be high, and we speculated that it is difficult to accurately evaluate the efficacy of ssBAE for long-term haemostasis in such patients (figure 1). We collected data on all the variables shown in tables 1 and 2 retrospectively from the patient records. The outcome data were collected using a variety of methods, including a review of patient records, phone conversation records between EHPC staff members and patients or family members, communication with other hospitals where the patients received follow-up care, or follow-up questionnaires sent from the EHPC to the participants. This
Figure 1 Patient selection flow. SSBAE, super selective bronchial artery embolisation.

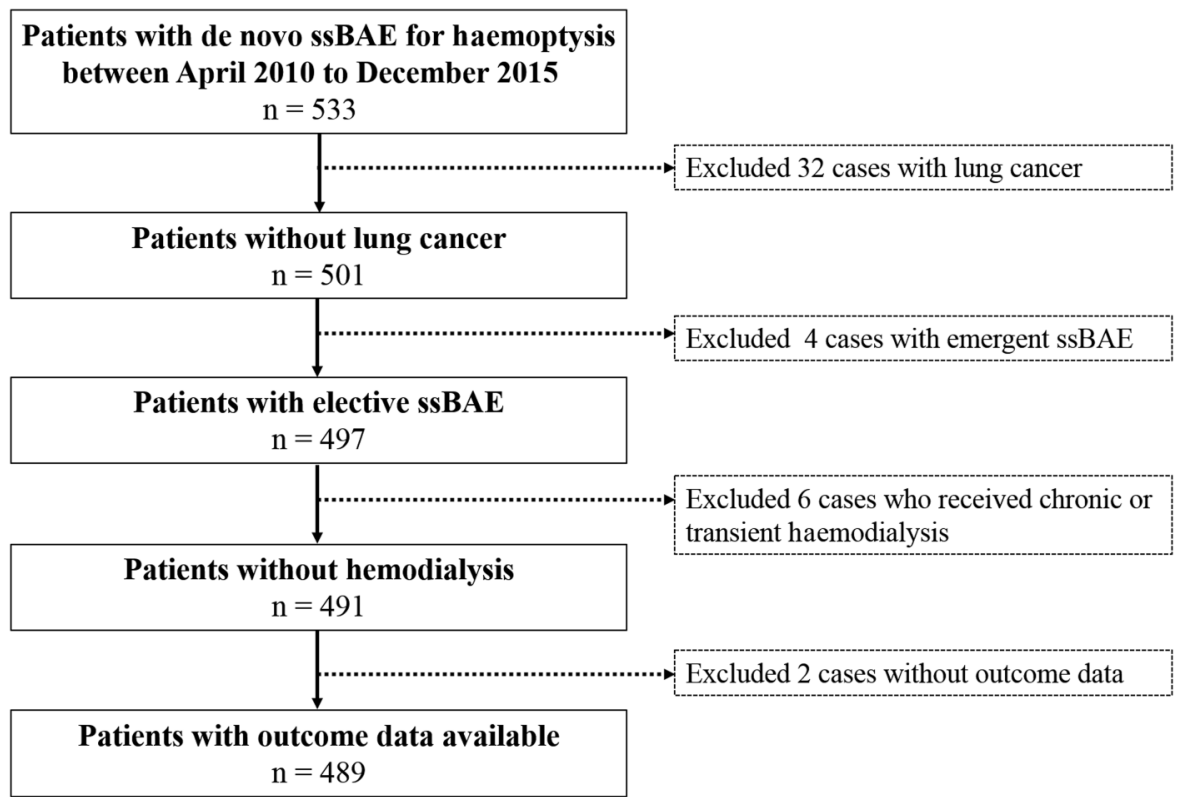


Table 1 Patient characteristics

\begin{tabular}{llr}
\hline Parameters & Total $(\mathbf{n}=489)$ & Missing \\
\hline Age (years) & $69(62-75)$ & 0 \\
Male (\%) & $227(46.4)$ & 0 \\
Body mass index $\left(\mathrm{kg} / \mathrm{m}^{2}\right)$ & $19.9(17.6-22.0)$ & 6 \\
Current smoker $(\%)$ & $54(13.2)$ & 81 \\
Baseline disease & & 0 \\
$\quad$ Bronchiectasis (\%) & $166(34.0)$ & \\
NTM (\%) & $115(23.5)$ & \\
Cryptogenic haemoptysis & $90(18.4)$ & \\
(\%) & & \\
$\quad$ Pulmonary aspergillosis (\%) & $65(13.3)$ & \\
Pulmonary Tb sequelae (\%) & $33(6.8)$ & \\
$\quad$ Other (\%) & $20(4.1)$ & \\
Laboratory data & & \\
$\quad$ Creatinine (mg/dL) & $0.67(0.56-0.81)$ & 1 \\
Haemoglobin (g/dL) & $12.5(11.2-13.5)$ & 0 \\
\hline NTM, non-tuberculous mycobacterium; Tb, tuberculosis. & \\
&
\end{tabular}

Table 2 Procedural Information

\begin{tabular}{lcl}
\hline Parameters & $\begin{array}{l}\text { Total } \\
\text { (n=489) }\end{array}$ & Missing \\
\hline Number of ssBAE sessions & & 0 \\
$1(\%)$ & $288(58.9)$ & \\
$2(\%)$ & $195(39.9)$ & \\
$3(\%)$ & $6(1.2)$ & 0 \\
Approach & $251(51.3)$ & \\
Femoral only (\%) & $5(1.0)$ & \\
Radial only (\%) & $233(47.7)$ & \\
Both (\%) & $186(38.0)$ & \\
Separately (\%) & $47(9.6)$ & \\
Simultaneously (\%) & $4(2-7)$ & 0 \\
Total number of target vessels & $489(100)$ & 0 \\
First ssBAE session & $3(2-5)$ & 0 \\
Number of target vessels (\%) & $450(92.0)$ & 0 \\
Success rate (\%) & $201(41.1)$ & 0 \\
Second ssBAE session & $3(2-4)$ & 0 \\
Number of target vessels (\%) & $194(96.5)$ & 0 \\
Success rate (\%) & $6(1.2)$ & 0 \\
Third ssBAE session & $2(2-2)$ & 0 \\
Number of target vessels (\%) & $6(100)$ & 0 \\
Success rate (\%) & $2(0.4)$ & 0 \\
Use of N-butyl-2-cyanoacrylate (\%) & $20(4.1)$ & 0 \\
Use of gelatin sponge (\%) & $8(1.6)$ & 0 \\
Complications in total (\%) & $1(0.2)$ & \\
Aortic dissection (\%) & $2(0.4)$ & \\
Symptomatic cerebral or & & \\
cerebellar infarction (\%) & $0(0.0)$ & \\
Spinal ischaemia (\%) & $5(1.0)$ & \\
Mediastinal haematoma (\%) & & \\
ssBAE, super selective bronchial artery embolisation. & \\
& &
\end{tabular}

study was supported by an independent Boston Scientific Corporation Investigator-Initiated Research Grant. Informed consent was obtained through the questionnaires. However, the requirement of written informed consent was waived because this study used retrospective data obtained mainly from the hospital records. Several cases have previously been published as case reports in another journal, and we obtained reprint permissions for the modified use of images from the journal (all images were modified and different from the original images). ${ }^{16}$

\section{Identification of haemoptysis-related arteries}

All of the patients underwent CT angiography with contrast medium enhancement before the procedure to evaluate the underlying causes of the haemoptysis, site of bleeding and possible haemoptysis-related arteries (HRAs) for procedural planning using 16-row multidetector CT (SIEMENS SOMATOM Emotion 16). ${ }^{5} 6917$ The scan parameters were as follows: collimation $1.6 \times 1.2 \mathrm{~mm}$; tube voltage $130 \mathrm{kV}$; the tube current was determined by the CARE Dose4D system according to the patient's physique and ranged from 20 to $345 \mathrm{~mA}$; rotation time $600 \mathrm{~ms}$; pitch $1.2 \mathrm{~mm}$; slice thickness $1.5 \mathrm{~mm}$ and reconstructed increment $0.70 \mathrm{~mm}$. We tried to determine all possible HRAs with the CT angiography screening by using three-dimensional reconstructed images (figure 2). The three-dimensional images were reconstructed using $1.5 \mathrm{~mm}$ diameter slices, using the Ziostation 2 classic S V.2.0.1.0 (Zio soft, Tokyo, Japan). All of the CT angiography screenings were performed by highly experienced radiation technologists in a comprehensive manner. We identified several previously reported findings as signs of possible HRA as shown in figure 2. These consisted of dilation of the vessels as compared with the normal size for that site, tortuosity of the vessels, direct shunting of the vessels, aneurysmal formation, pleural adhesion with intercostal artery invasion into the lungs and ground glass opacity, suggesting inhaled blood, although some of these signs were only identified in select cases. ${ }^{15} 689^{9}$ The candidate HRAs included arteries related to the (1) aorta, for example, bronchial, intercostal, pulmonary ligament and inferior phrenic arteries; (2) subclavian artery, for example, internal thoracic, supreme intercostal and inferior thyroid arteries; and (3) axillary artery, for example, lateral thoracic, thoracoacromial and dorsal scapular arteries. ${ }^{15} 689$

\section{Standard protocol of SsBAE}

All of the ssBAE procedures were performed by highly experienced interventional pulmonologists, who are respiratory physicians undertaking at least 50 interventional radiology procedures annually. Our standard ssBAE protocol required two approach routes. The ssBAE for the aorta-related HRAs was performed via a transfemoral approach, and that for the subclavianrelated and axillary-related HRAs was performed via a transradial or transbrachial approach. The number and approach sites of the ssBAE sessions were determined on the basis of the attending physician's discretion, after taking into consideration the condition of the patient and the possible HRAs suggested by the CT findings. All 

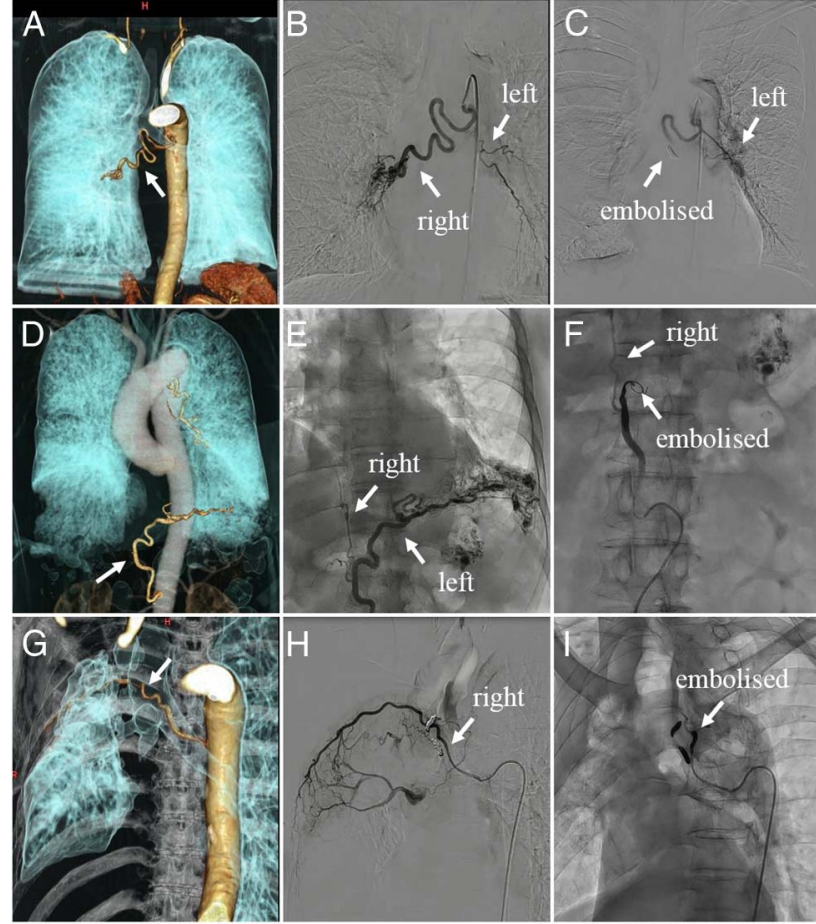

Figure 2 Three-dimensional CT and angiographic findings of HRA before and after ssBAE. Three-dimensional CT (A, D, G) and angiographic findings of HRA before $(B, E, H)$ and after $\operatorname{ssBAE}(\mathrm{C}, \mathrm{F}, \mathrm{I})$. The uppercase letters $(\mathrm{A}-\mathrm{C})$ indicate an abnormal right $\mathrm{BA}$ and normal left $\mathrm{BA}$ with a common trunk, and the abnormal right BA was successfully embolised with three platinum coils after the administration of a gelatin sponge (C). The middle case (D-F) demonstrates abnormal left IPA and normal right IPA with a common trunk, and the abnormal left IPA was successfully embolised with three platinum coils $(F)$. The lowercase letters $(G-I)$ indicate an abnormal right fifth IA, which was embolised with three platinum coils after the administration of a gelatin sponge (I). All abnormal arteries showed dilated and tortuous anatomical deformations with hypervascularity and shunting of the pulmonary artery. Modified reproduction with permission from the Journal of Typical Medical Images and Videos ( $J$ Typ Med Images Video 2016: Case ID 147, 148 and 156, http:// thejtmiv.com, last accessed on 20 December 2016). BA, bronchial artery; HRA, haemoptysis-related artery; IA, intercostal artery; IPA, inferior phrenic artery; ssBAE, super selective bronchial artery embolisation.

possible HRAs identified with CT were super selectively evaluated using arteriography with 5 Fr guiding catheter and 3 Fr microcatheter systems with 0.014 or 0.016 -inch guide wires during the session. Aortography was not performed in general. When abnormal findings were observed, such as systemic-pulmonary shunts, proliferations of the capillary vessels or extravasation of the contrast medium to the lung tissues, they were super selectively embolised using the $3 \mathrm{Fr}$ microcatheter system. The embolic materials used in all cases were platinum detachable or pushable coils (IDC or Interlock or VortX Boston Scientific Japan, Target Stryker Japan, Nester Cook Japan, C-STOPPER PIOLAX). The standard coil deployment manoeuvre is shown in figure 3. We attempted to embolise each HRA by using $\leq 3$ platinum coils whenever possible, considering the insurance cost limitation in Japan. NBCA and gelatin sponges were used in several cases as a subsidiary treatment on a trial basis. Our speculation for these subsidiary treatments was that preocclusion with gelatin sponges may reduce the number of coils needed for embolisation or that postcoiling NBCAs may improve the long-term haemostatic effect after ssBAE. PVA was not used because it was not covered by the medical insurance in Japan during the study period. Procedural success was defined as successful embolisation of all target HRAs during the ssBAE sessions, and failure of even one HRA embolisation was considered a procedural failure.

\section{Statistical analysis}

The primary end point was the composite of the 3-year recurrence of haemoptysis and mortality from the day of the last ssBAE session. Secondary end points were the 3-year recurrence-free haemoptysis rate and 3-year survival rate. The patients who were alive were censored at the time of their last follow-up. Continuous variables were summarised using medians and IQRs (quartiles 1-3) and categorical variables using means of counts and percentages. The Kaplan-Meier analysis was employed to estimate the incidence of each end point, and the adjusted analysis of variance (ANOVA) was used to test the differences among the underlying diseases. The ANOVA was assessed using the multivariable Cox proportional hazard regression model. We used the following variables as covariates in the adjusted ANOVA with multiple imputation of missing variables: age, sex, body mass index, current smoking, ssBAE approach and total number of target vessels. All statistical tests were two-sided, with an $\alpha$ level of 0.05 considered statistically significant. All statistical analyses were performed using the R software packages (V.3.2.1; R Development Core Team).

\section{RESULTS}

In this study, 489 consecutive patients with a total of 696 ssBAE procedures were retrospectively analysed. The patient characteristics are summarised in table 1 . The median patient age was 69 years, and $46.4 \%$ were men. Table 2 details the procedural information. The number of ssBAE sessions was one in $58.9 \%$ of all study patients, two in $39.9 \%$ and three in $1.2 \%$. The average number of target vessels was $4(2-7)$. The total procedural success rate was $93.4 \%$, and the remaining $6.6 \%$ failed mainly due to one vessel embolisation failure because of the difficulty in embolising the ectopic bronchial artery derived from the underline part of the aortic arch. There were only $8(1.6 \%)$ major complications: 1 aortic dissection, 2 symptomatic cerebellar infarctions and 5 mediastinal haematoma cases. Aortic dissection occurred in a patient with abdominal aortic aneurysm during the 

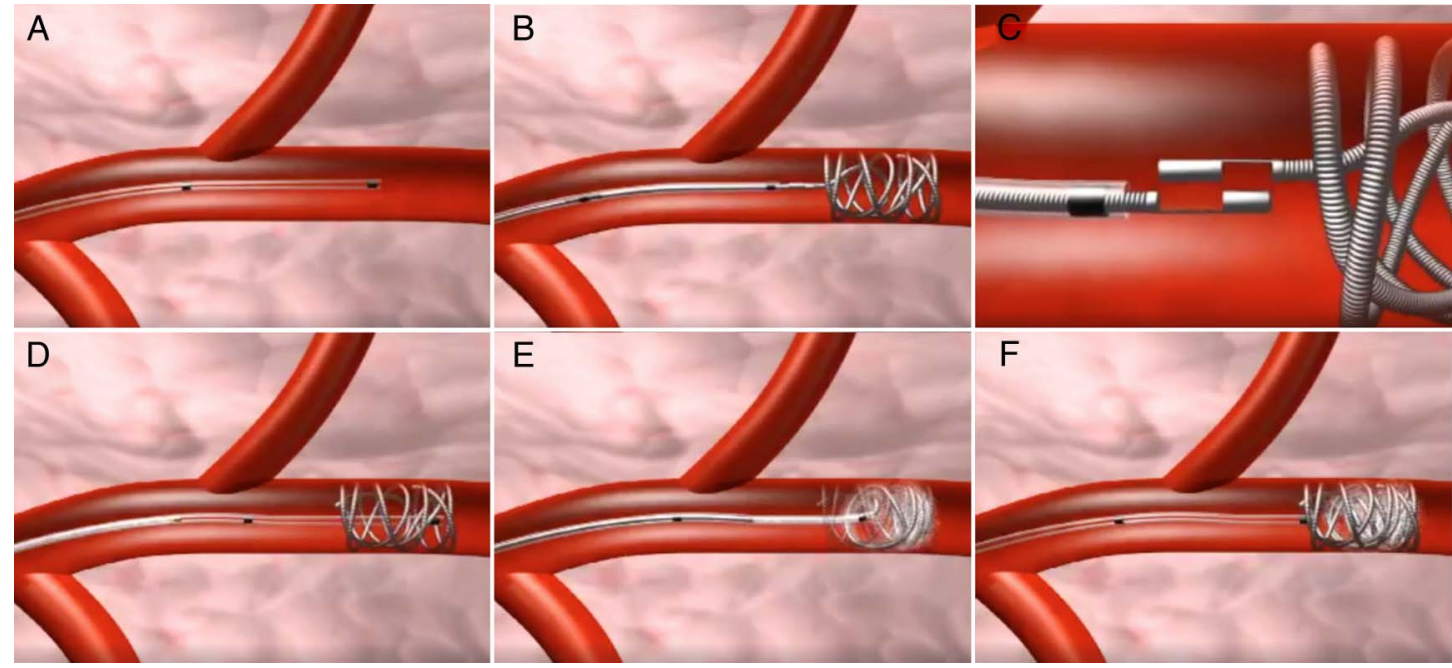

Figure 3 Schematic diagram of the standard coil deployment manoeuvre in bronchial artery embolisation. Our standard bronchial artery coil embolisation manoeuvre included two steps. First is anchoring coil deployment $(A-C)$ followed by filling or finishing coil deployment (D-F). After placing the microcatheter in the arterial area of embolisation (A), an IDC-18 soft interlocking detachable coil (Boston Scientific, Tokyo, Japan) was inserted as an anchoring coil (B). The detachable coil can be advanced and retracted before the final placement. The controlled coil deployment was achieved by isolating the interlocking arm (C). Then we carefully removed the delivery wire, and a similar procedure was performed again with the VortX Fibered pushable coil (Boston Scientific, Tokyo, Japan) as a filling or finishing coil. The purpose of the anchoring coil deployment was to gain a foothold for the following dense coiling, named filling or finishing coil deployment. We prefer the use of a highly thrombogenic coil as a filling or finishing coil. You can also refer to online supplementary video 1. More detailed information is also available on each product website.

guiding catheter manipulation for intercostal arteriography. We speculated that the main cause of this complication was aortic wall fragility. One case of cerebellar infarction occurred during the supreme intercostal arteriography via the femoral approach, possibly because of atheromatous debris embolisation in the vertebral artery derived from the tip of the catheter. This could be avoided with the transradial approach. The other case of cerebellar infarction occurred after the procedure; its possible mechanism included thrombus embolisation derived from the proximal part of the embolised supreme intercostal and internal mammary arteries. Thus, coil embolisation cases near the vertebral artery need careful attention. In addition, mediastinal haematoma was addressed by haemostasis with platinum coil embolisation of the proximal arteries. Figure 2 shows the representative cases with three-dimensional CT and angiographic findings of the HRA before and after ssBAE.

There were 57 cases of recurrent haemoptysis and 23 deaths in this study. Figure 4 details the Kaplan-Meier estimates for each end point. The haemoptysis-free survival rates were $86.9 \%(95 \%$ CI $83.7 \%$ to $90.2 \%)$ at 1 year, $79.4 \%(74.8 \%$ to $84.3 \%)$ at 2 years and $57.6 \%$ $(45.1 \%$ to $73.4 \%)$ at 3 years. Five deaths were associated with haemoptysis, five were due to the exacerbation of underlying diseases, five due to pneumonia and eight due to unknown causes. Although it was not statistically significant, cryptogenic haemoptysis tended to show the most favourable outcome, while non-tuberculous mycobacterium (NTM) had the worst outcome (adjusted ANOVA $\mathrm{p}=0.250$ ). The recurrence-free haemoptysis rates were $90.4 \%(87.6 \%$ to $93.3 \%)$ at 1 year, $85.9 \%(82.1 \%$ to $89.9 \%)$ at 2 years and $67.7 \%$ (55.5\% to $82.6 \%)$ at 3 years. The survival rates were $96.2 \%(94.3 \%$ to $98.0 \%)$ at 1 year, $93.3 \%$ (90.3\% to $96.4 \%)$ at 2 years and $91.8 \%(88.2 \%$ to $95.5 \%)$ at 3 years.

\section{DISCUSSION}

In this study, we demonstrated the safety and long-term efficacy of elective ssBAE using platinum coils in a comprehensive manner by enrolling 489 patients with 696 procedures. ${ }^{10-15}$ The large sample size and standardised protocol for the elective ssBAE allowed us to clearly evaluate the influence of ssBAE, using platinum coils, on persistent haemoptysis. The safety and long-term efficacy of ssBAE using platinum coils were comparable with the results achieved using other materials for ssBAE, such as PVA or NBCA. ${ }^{10-14}$ Since only a little evidence on ssBAE using platinum coils was previously available, our study provides physicians with new insights into this field. ${ }^{15}$ We believe that our data will contribute to advancements in the treatment of patients with haemoptysis which severely impairs the patients' quality of life.

\section{Coil embolisation}

The rationale for using $\mathrm{BAE}$ is that one of the main causes of haemoptysis is the placement of a systemic-pulmonary shunt. Four available materials are typically used 
Figure 4 Kaplan-Meier estimate

of each end point. * $p$ Value with adjusted ANOVA. The

parentheses indicate the number of patients at risk. ANOVA, analysis of variance; NTM, non-tuberculous mycobacterium; $\mathrm{Tb}$, tuberculosis.
A Haemoptysis-free Survival

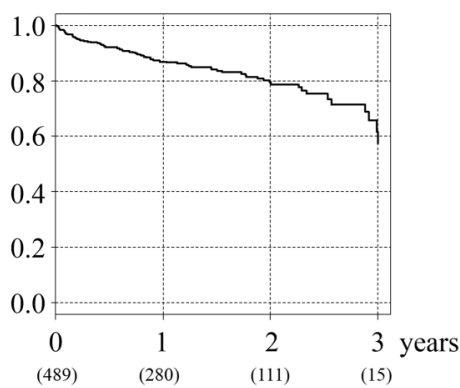

B Haemoptysis-free Survival

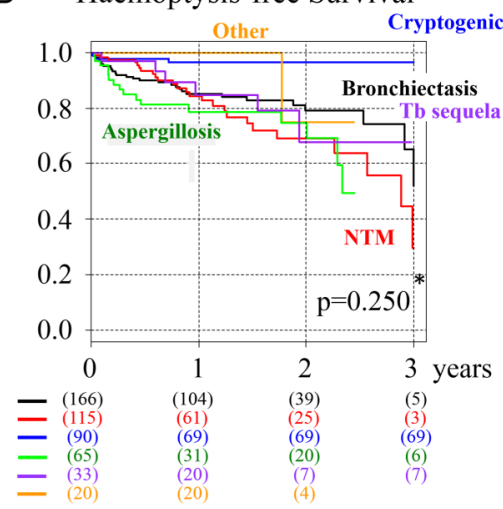

D

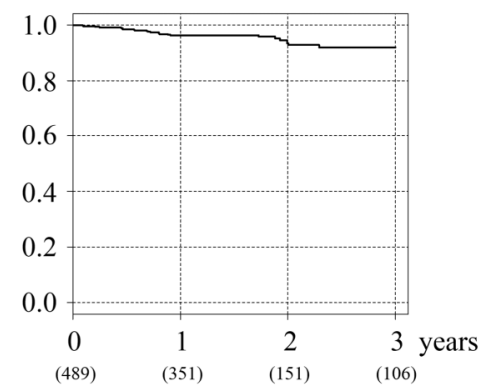

for embolising HRAs. ${ }^{4-8}$ The most conventional and popular embolisation material is the gelatin sponge. It is still often employed since it is quite inexpensive and easy to use; however, its effectiveness is usually limited and temporal owing to resorption. ${ }^{5}{ }^{612}$ To overcome this limitation, PVA and NBCA have been introduced. In particular, the long-term efficacy of NBCA is impressive. ${ }^{13}{ }^{14}$ However, it should be emphasised that extreme caution should be exercised when using liquid embolisation materials, such as NBCA, owing to the risk of misembolisation, particularly in the spinal branches, if the interventionist is not sufficiently experienced. ${ }^{5} 613141819$

In contrast, the unique aspect of our standard protocol for ssBAE is that we used platinum coils for the embolisation material. ${ }^{15}$ At this point, platinum coils are not typically used for BAE; they are used most often in very specific situations, such as pulmonary arteriovenous malformations or Rasmussen aneurysms. ${ }^{20}{ }^{21}$ While all embolisation materials have their pros and cons, we have found that using platinum coils makes it relatively easy to control the site of embolisation, when compared with blood flow dependent-type materials. ${ }^{5}{ }^{6}$ In fact, our total procedural success rate was $93.4 \%$ with only a $1.6 \%$ major complication rate, suggesting a consistent, secure and controlled coil implantation. These excellent procedural results were partly because of the very high visibility of the platinum coils under fluoroscopic guidance. Although some authors have reported that metallic coil embolisation can occlude more proximal vessels and may preclude repeat embolisation if haemoptysis recurs, ${ }^{5} 62021$ we can perform ssBAE repeatedly to embolise HRAs just proximal to the previously occluded site when haemoptysis recurs (refer to the remaining proximal vessels of the HRAs in figure $2 \mathrm{C}, \mathrm{F}, \mathrm{I})$. We believe that compared with the linear liquid embolisation of bloodstream-dependent materials, pinpoint embolisation of platinum coils contributes to the controllable HRA embolisation. Detachable coils are a good option for inexperienced interventional pulmonologists, whereas liquid-type material embolisation cannot be retried. Further, authors especially find the utility of detachable coils in cases where the backup force of guiding catheters is insufficient. In this case, it is difficult to deploy the coil in the target site because the position of the microcatheter cannot be fixed easily, and retractions may be needed several times. Even though it is difficult to discuss issues on the cost-effectiveness of ssBAE in detail because the cost and standard procedures may be different among institutions, the primary disadvantage of metallic coils for ssBAE is their cost, especially with detachable coils. However, this problem could be resolved with the increased use of pushable platinum coils for ssBAE by experienced interventionists, since the cost is one-fourth that of detachable coils. Cost-effectiveness, as well as the risks and benefits, should be determined by each physician depending on their experiences.

\section{Long-term prognosis}

In this study, we showed that the haemoptysis-free survival rates were $86.9 \%(83.7 \%$ to $90.2 \%)$ at 1 year, $79.4 \%$ 
$(74.8 \%$ to $84.3 \%)$ at 2 years and $57.6 \%(45.1 \%$ to $73.4 \%)$ at 3 years. Even though we should interpret these data with caution since patient prognoses may differ depending on their underlying diseases, the procedural outcomes were favourable and comparable with those of other ssBAE materials, such as PVA or NBCA. ${ }^{10-14}$ For example, Woo $e a^{14}$ reported that the 1-year and 3-year haemoptysis-free survival rates of ssBAE with PVA $(\mathrm{n}=293)$ were $77 \%$ and $68 \%$, respectively, and those with NBCA $(n=113)$ were $88 \%$ and $85 \%$, respectively, in one of the largest retrospective observational studies.

We also determined that cryptogenic haemoptysis tended to show the most favourable outcome, whereas NTM tended to show the worst outcome, although these differences were not statistically significant. These findings were consistent with those of previous experimental reports, which suggested prognostic differences for haemoptysis depending on the underlying diseases. For example, several studies reported worse outcomes in patients with chronic tuberculosis or aspergillosis. ${ }^{5} 101114$

Recurrence of haemoptysis after BAE can be caused pathomechanically by incomplete embolisation, recanalisation of the embolised bronchial artery, collateralisation by other arterial vascular territories, missed culprit vessel identification, inadequate causal therapy or progression of the underlying pulmonary disease. ${ }^{5}{ }^{6}$ Evaluation of the mechanisms of recurrent haemoptysis after ssBAE using metallic coils and identification of preventive strategies for recurrent haemoptysis are additional clinical questions that require further studies.

At any rate, selective coil embolisation should be considered as one of the first-line options for haemoptysis rather than being viewed as inferior to particle embolisation considering its long-term efficacy and safety to avoid non-target tissue necrosis owing to the controllability of embolisation of detachable coils.

\section{Study limitations}

There are several study limitations that should be mentioned. First, it was difficult to objectively evaluate the amount of haemoptysis retrospectively because data on the severity of haemoptysis were not collected in a uniform quantitative manner. ${ }^{3}$ Second, the control of the patients' underlying respiratory disorders also influenced their prognosis. Since many of the study patients were medically managed by attending pulmonologists at outside facilities and were referred to our institution specifically to undergo elective ssBAE, detailed information about the status of their respiratory disorders was not available in this study. ${ }^{6}$ Third, there are criticisms that non-massive haemoptysis should not be treated using ssBAE because some physicians express concern about the safety and lack of evidence of its long-term efficacy. However, we believe that attending pulmonologists can understand how sudden unexpected haemoptysis impairs the patients' quality of life even though it is non-massive. Attending physicians should understand how the extrication from persistent unexpected haemoptysis can be valuable for these patients. All patients in this study accepted the risk of major complications. Fourth, some procedure-related information, such as procedural time, was not available during the study period, although the median procedural time was 113 min recently. Fifth, the definition of major complications varies among studies; however, we employed the definition from the guideline of the Society of Interventional Radiology Standards of Practice Committee. ${ }^{22}$ Finally, our study is a single-centre, retrospective observational study. Our data should be interpreted considering these limitations.

\section{CONCLUSIONS}

We demonstrated the safety and long-term efficacy of elective ssBAE using platinum coils. ssBAE using platinum coils can be a valuable therapeutic option for treating patients with haemoptysis.

\section{Author affiliations}

${ }^{1}$ Hemoptysis and Pulmonary-Circulation Center, Kishiwada Eishinkai Hospital, Kishiwada, Japan

${ }^{2}$ Department of Clinical Epidemiology and Biostatics, Osaka University Graduate School of Medicine, Suita, Japan

${ }^{3}$ Department of Radiology, National Hospital Organization Kinki-Chuo Chest Medical Center, Sakai, Japan

${ }^{4}$ Rakuwakai Kyoto Pulmonary Center, Rakuwakai Otowa Hospital, Kyoto, Japan

Acknowledgements The authors express their gratitude to Dr Seiei Shirakawa, the president of Kishiwada Eishinkai Hospital, Unshow Shirakawa, the vice president, and the Japan Society of Clinical Research for their dedicated support for our works.

Contributors $\mathrm{HI}, \mathrm{MH}$ and MR had full access to all of the study data and take responsibility for the integrity of the data and accuracy of the data analysis, including and especially any adverse effects. HI, MH, MR, JT, MY, MA, YN, DK, KY and AS contributed substantially to the study design, data analysis and interpretation, and to the writing and final approval of the manuscript.

Funding This study was supported by an independent Boston Scientific Corporation Investigator-Initiated Research Grant.

\section{Competing interests None declared.}

Ethics approval The study protocol complied with the Helsinki Declaration standards and was approved by the Ethical Committee of the EHPC (approval number 2012-02).

Provenance and peer review Not commissioned; externally peer reviewed.

Data sharing statement No additional data are available.

Open Access This is an Open Access article distributed in accordance with the Creative Commons Attribution Non Commercial (CC BY-NC 4.0) license, which permits others to distribute, remix, adapt, build upon this work noncommercially, and license their derivative works on different terms, provided the original work is properly cited and the use is non-commercial. See: http:// creativecommons.org/licenses/by-nc/4.0/

\section{REFERENCES}

1. Swanson KL, Johnson CM, Prakash UB, et al. Bronchial artery embolization: experience with 54 patients. Chest 2002;121: 789-95.

2. Govind M, Maharajh J. The impact of coinfection with human immunodeficiency virus and pulmonary tuberculosis on the success of bronchial artery embolization. Br J Radiol 2013;86:20120256. 
3. Lee MK, Kim SH, Yong SJ, et al. Moderate hemoptysis: recurrent hemoptysis and mortality according to bronchial artery embolization. Clin Respir J 2015;9:53-64.

4. Poyanli A, Acunas B, Rozanes I, et al. Endovascular therapy in the management of moderate and massive haemoptysis. $\mathrm{Br} \mathrm{J}$ Radiol 2007;80:331-6.

5. Yoon W, Kim JK, Kim YH, et al. Bronchial and nonbronchial systemic artery embolization for life-threatening hemoptysis: a comprehensive review. Radiographics 2002;22:1395-409.

6. Ittrich $\mathrm{H}$, Klose $\mathrm{H}$, Adam $\mathrm{G}$. Radiologic management of haemoptysis: diagnostic and interventional bronchial arterial embolisation. Rofo 2015;187:248-59.

7. Remy J, Arnaud A, Fardou H, et al. Treatment of hemoptysis by embolization of bronchial arteries. Radiology 1977;122:33-7.

8. Vujic I, Pyle R, Hungerford GD, et al. Angiography and therapeutic blockade in the control of hemoptysis. The importance of nonbronchial systemic arteries. Radiology 1982;143:19-23.

9. Bruzzi JF, Rémy-Jardin M, Delhaye D, et al. Multi-detector row CT of hemoptysis. Radiographics 2006;26:3-22.

10. Kato A, Kudo S, Matsumoto K, et al. Bronchial artery embolization for hemoptysis due to benign diseases: immediate and long-term results. Cardiovasc Intervent Radiol 2000;23:351-7.

11. Chun JY, Belli AM. Immediate and long-term outcomes of bronchial and non-bronchial systemic artery embolisation for the management of haemoptysis. Eur Radiol 2010;20:558-65.

12. Hahn S, Kim YJ, Kwon W, et al. Comparison of the effectiveness of embolic agents for bronchial artery embolization: gelfoam versus polyvinyl alcohol. Korean J Radiol 2010;11:542-6.

13. Yoo DH, Yoon CJ, Kang SG, et al. Bronchial and nonbronchial systemic artery embolization in patients with major hemoptysis: safety and efficacy of N-butyl cyanoacrylate. AJR Am J Roentgenol 2011;196:W199-204.

14. Woo S, Yoon CJ, Chung JW, et al. Bronchial artery embolization to control hemoptysis: comparison of N-butyl-2-cyanoacrylate and polyvinyl alcohol particles. Radiology 2013;269:594-602.

15. Okuda K, Masuda K, Kawashima M, et al. Bronchial artery embolization to control hemoptysis in patients with Mycobacterium avium complex. Respir Investig 2016;54:50-8.

16. Ishikawa H. J Typ Med Images Video 2016: Case ID 147, 148 and 156. http://thejtmiv.com (accessed 20 Dec 2016).

17. Abal AT, Nair PC, Cherian J. Haemoptysis: aetiology, evaluation and outcome-a prospective study in a third-world country. Respir Med 2001;95:548-52.

18. Schrodt JF, Becker GJ, Scott JA, et al. Bronchial artery embolization: monitoring with somatosensory evoked potentials. Work in progress. Radiology 1987;164:135-9.

19. Brown AC, Ray CE. Anterior spinal cord infarction following bronchial artery embolization. Semin Intervent Radiol 2012;29: 241-4

20. Remy J, Smith M, Lemaitre L, et al. Treatment of massive hemoptysis by occlusion of a Rasmussen aneurysm. AJR Am $J$ Roentgenol 1980;135:605-6.

21. Pollak JS, Saluja S, Thabet A, et al. Clinical and anatomic outcomes after embolotherapy of pulmonary arteriovenous malformations. J Vasc Interv Radiol 2006;17:35-44.

22. Angle JF, Siddiqi NH, Wallace MJ, et al. Society of Interventional Radiology Standards of Practice Committee. Quality improvement guidelines for percutaneous transcatheter embolization: Society of Interventional Radiology Standards of Practice Committee. J Vasc Interv Radiol 2010;21:1479-86. 